\title{
Convergence analysis for Turbo-equalization
}

\author{
Aline Roumy ${ }^{1,2}$, Alex J. Grant ${ }^{3}$, Inbar Fijalkow ${ }^{2}$, Paul D. Alexander ${ }^{4}$, Didier Pirez ${ }^{5}$ \\ ${ }^{1}$ Dept. of Electrical Engineering, Princeton University, Princeton, NJ 08544, USA, \\ ${ }^{2}$ Equipe de Traitement des Images et du Signal, ENSEA-University of Cergy-Pontoise, 95014 Cergy, France, \\ ${ }^{3}$ Institute for Telecommunications Research, University of South Australia, Mawson Lakes, SA 5095, Australia, \\ ${ }^{4}$ Southern Poro Communications Annandale, NSW 2038, Australia, \\ ${ }^{5}$ THOMSON-CSF Communications, 92231 Gennevilliers Cedex, France.
}

In high rate communication, where the transmitted signal is subject to intersymbol interference (ISI), we may use equalization to reduce the effect of ISI and channel coding to correct any remaining errors. A conventional equalizer does not make use of the redundancy introduced by the channel coding: equalization and decoding are disjoint which is not optimal in the sense of the minimization of the error probability. Since optimal joint equalization and decoding is an NP-complete problem, we consider a relevant trade-off between complexity and performance: that is the Turbo-equalizer, first proposed in [4] and analyzed in [7]. Our goal is to study the evolution of the effective error variance through the iterative process involved in the Turbo-equalizer by following the approach described in [1]. This may also allow prediction of the performance and give insight into observed phenomena for the Turbo-equalizer.

\section{Turbo-Equalizer}

Consider the transmitter described in Figure 1. The discrete channel is characterized by its impulse response $h_{n}$. So, the samples of the received signal can be written as $r_{n}=h \star d_{n}+w_{n}$, where $\star$ stands for the convolution and $w_{n}$ is a white gaussian noise.

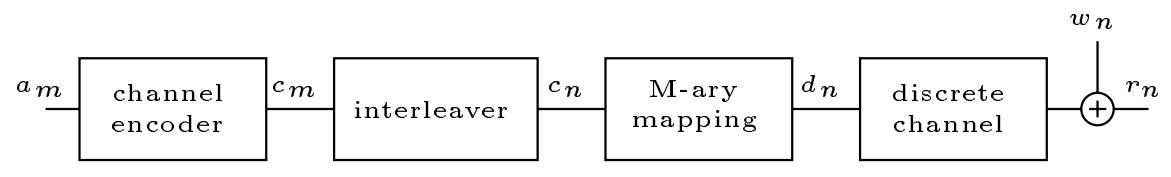

Fig. 1. Transmitter.

The Turbo-Equalizer is well fitted to this transmitter and is implemented in a modular pipelined structure with $P$ identical iterations. With reference to Figure 2, each module consists of the concatenation of a Soft Input/Soft Output equalizer, a soft de-mapper (symbol to bit), a deinterleaver, an APP decoder, an interleaver and a soft re-mapper (bit to symbol). The output of iteration $p$ together with the channel output is input to iteration $p+1, p=1,2, \cdots, P$. Decoding is carried out by using the APP computer [2]. Given the observation and prior probabilities, the decoder computes the marginal log-likelihood of the coded bits in the form of $\tilde{\Lambda}\left(c_{m}\right)=\ln \left(\frac{P\left(c_{m}=1 \mid \mathbf{y}\right)}{P\left(c_{m}=0 \mid \mathbf{y}\right)}\right)$. After deinterleaving, an estimate $d_{n}^{p}$ of the conditional mean value of the symbols $d_{n}$ is calculated as in [4]. In the case of binary modulation (BPSK): $d_{n}^{p}=$ $\mathcal{P}\left(c_{m}=1 \mid \mathbf{y}\right)-\mathcal{P}\left(c_{m}=0 \mid \mathbf{y}\right)$. 


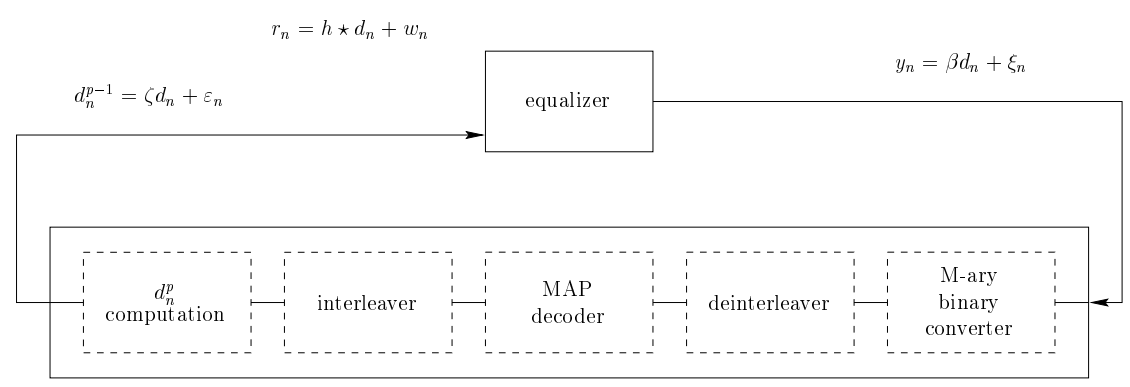

Fig. 2. Module $p$ of the Turbo-equalizer.

As shown in Figure 3, the equalizer used here is the Interference Canceler (IC). It consists of two filters $P(z)$ and $Q(z)$ and is fed by both the channel output, $r_{n}$ and the output of the previous module, $d_{n}^{p-1}$. It therefore takes into account the redundancy of the coded symbols transmitted over the time dispersive channel. Note that the equalizer of the very first iteration is a Decision Feedback Equalizer (DFE) that just processes the output of the channel because the prior symbol averages are $d_{n}^{0}=0,0, \cdots, 0$. Let us

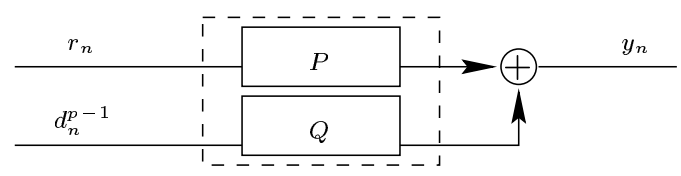

Fig. 3. Equalizer in the module $p>1$.

denote the transfer function of the discrete channel as $H(z)$. Minimization of the mean square error MSE $=E\left[\left|y_{n}-d_{n}\right|^{2}\right]$ over the coefficients of the filters $P$ and $Q$ under the constraint $q_{0}=0$, when $Q$ is fed by the $d_{n}$, yields the filters in the form [4]:

$$
P(z)=\alpha H^{*}\left(z^{-1 *}\right), Q(z)=\alpha\left(H(z) H^{*}\left(z^{-1 *}\right)-\gamma_{h}(0)\right)
$$

where $H(z)=\sum_{i} h_{i} z^{-i}, H^{*}\left(z^{-1 *}\right)=\sum_{i} h_{i}^{*} z^{i}, \gamma_{h}(0)=\sum_{i}\left|h_{i}\right|^{2} \alpha=\frac{\sigma_{d}^{2}}{\sigma_{d}^{2} \gamma_{h}(0)+\sigma_{w}^{2}}$. ${ }^{*}$ stands for conjugation whereas $\sigma_{d}^{2}$ and $\sigma_{w}^{2}$ stand respectively for the transmitted symbol power and thermal noise power.

$P$ is the filter matched to the channel $H$ and $Q+\alpha \gamma_{h}(0)$ its autocorrelation. $Q$ is used to remove the ISI from the present estimate caused by previous and future detected symbols. We have shown earlier [7] that it results in complete elimination of ISI, provided that the previous and future decisions are correct.

\section{Convergence Analysis}

Note that the Turbo-equalizer presented above gives an estimate of the transmitted symbol $d_{n}$ in two places: the equalizer output $y_{n}$ and decoder output $d_{n}^{p}$. Let us now split the Turbo-equalizer in two blocks and write the input and output of each block explicitly as estimates of $d_{n}$. This leads to the scheme shown in Figure 2. For tractable analysis, we follow the approach described in [1] and represent the Turbo iteration as the evolution of error variances on $d_{n}$. In the following, we approximate the distribution of these estimates given $d_{n}$ by a white gaussian one. Note that under this white gaussian assumption, the error is fully characterized by its variance. 


\section{A. Model and principle}

The Turbo-equalizer is fed by the output of the channel:

$$
r_{n}=h \star d_{n}+w_{n}, \text { with normalized thermal noise variance } \sigma_{w, N}^{2}=\frac{\sigma_{w}^{2}}{\gamma_{h}(0) \sigma_{d}^{2}},
$$

and the estimates of $d_{n}$ are either

$$
d_{n}^{p-1}=\zeta d_{n}+\varepsilon_{n}, \text { with effective normalized error variance } \sigma_{\varepsilon, N}^{2}=\frac{\sigma_{\varepsilon}^{2}}{\zeta^{2} \sigma_{d}^{2}},
$$

or

$$
y_{n}=\beta d_{n}+\xi_{n}, \text { with effective normalized error variance } \sigma_{\xi, N}^{2}=\frac{\sigma_{\xi}^{2}}{\beta^{2} \sigma_{d}^{2}},
$$

where $N$ stands for normalized.

Considering input variances $\sigma_{w, N}^{2}$ and $\sigma_{\varepsilon, N}^{2}$ to the IC block, we compute the output error variance $\sigma_{\xi, N}^{2}$. Under the assumptions that $\zeta=1$ and that $w_{n}$ and $\varepsilon_{n}$ are independent,

$$
\sigma_{\xi, N}^{2}=g_{\sigma_{w, N}^{2}}\left(\sigma_{\varepsilon, N}^{2}\right)=\sigma_{w, N}^{2}+\frac{\Gamma_{h}}{\gamma_{h}(0)^{2}} \sigma_{\varepsilon, N}^{2},
$$

where $\Gamma_{h}$ is obtained from the autocorrelation of the autocorrelation of the channel $h_{n}$ where the central term is suppressed:

$$
\Gamma_{h}=\sum_{n} \gamma_{h}(n) \gamma_{h}(-n)^{*}-\gamma_{h}(0)^{2} \delta_{n},
$$

which is a measure of dispersion.

The decoder updates the error variance $\sigma_{\varepsilon, N}^{2}$ via the function $f$ :

$$
\sigma_{\varepsilon, N}^{2}=f\left(\sigma_{\xi, N}^{2}\right)
$$

$f$ may be obtained through simulation, or bounded. Analytical characterization of $f$ is difficult and we concentrate on understanding the Turbo iteration, given $f$ via simulation.

\section{B. "Easy" channels}

We can now test the Turbo-equalizer convergence by plotting the output variance of the decoder $\sigma_{\varepsilon, N}^{2}$ versus the input variance $\sigma_{\xi, N}^{2}$ and the input variance of the equalizer (IC) $\sigma_{\varepsilon, N}^{2}$ versus the output variance $\sigma_{\xi, N}^{2}$ for a given thermal noise variance $\sigma_{w, N}^{2}$ (see Figure 4). This leads to the recurrence:

$$
\sigma_{\varepsilon, N}^{2 p+1}=f \circ g_{\sigma_{w, N}^{2}}\left(\sigma_{\varepsilon, N}^{2 p}\right)
$$

We are interested in the location and stability of fixed points of $f \circ g_{\sigma_{w, N}^{2}}$ and its stability. Given a fixed point $x$, the condition for stability is:

$$
\left|\left(f \circ g_{\sigma_{w, N}^{2}}\right)^{\prime}(x)\right|<1
$$

which depends indeed on $\sigma_{w, N}^{2}$. 


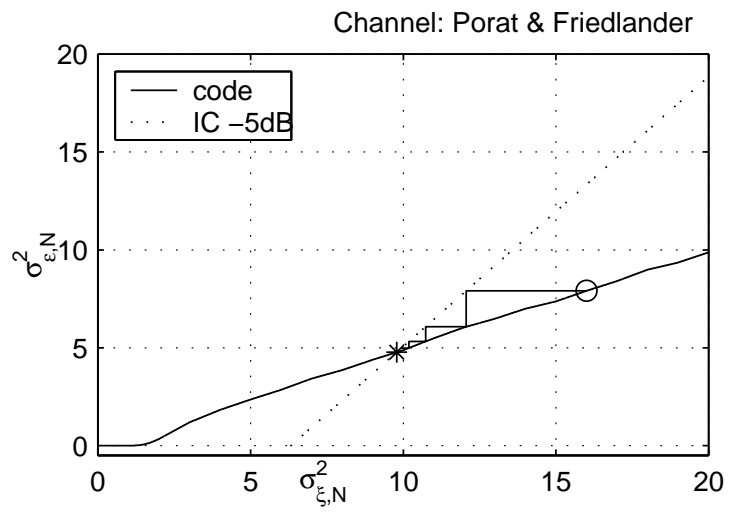

a. Fixed point: existence and convergence

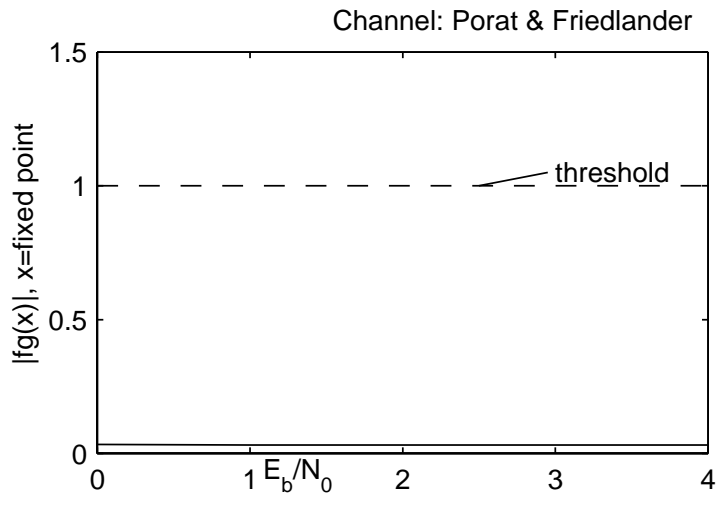

b. Stability of the fixed point

Fig. 4. Iterative process of the Turbo-equalizer (Porat and Friedlander's channel).

In practice, we have observed the existence of a stable fixed point for "easy" channels, that is to say dispersive channels with the same minimal distance as the AWGN channel (for instance Porat and Friedlander's channel [5] in Figure 4). We use here a 64-state recursive systematic code [133,171]. Moreover simulation shows that the Turbo-equalizer tends to the performance of the coded sequence tranmitted over AWGN channel at high SNR but not at low SNR (see Figure 5.a). This can be easily explained with the convergence analysis (see Figure 5.b). Given a noise variance $\sigma_{w, N}^{2}$, the decoder gives an output error variance plotted by + . As for the Turbo-equalizer, it tends to the fixed point $*$, which leads to an extra variance $\Delta$ for the Turbo-equalizer at high $\sigma_{w}^{2}$. Note that the smaller the slope of $g_{\sigma_{w, N}^{2}}^{-1}$ (inverse of $g_{\sigma_{w, N}^{2}}$ ) is, the bigger the difference is and the tougher the channel is. So we can define a "tough" channel, when processed by the Turbo-equalizer, as a channel with small $\frac{\gamma_{h}(0)^{2}}{\Gamma_{h}}$.

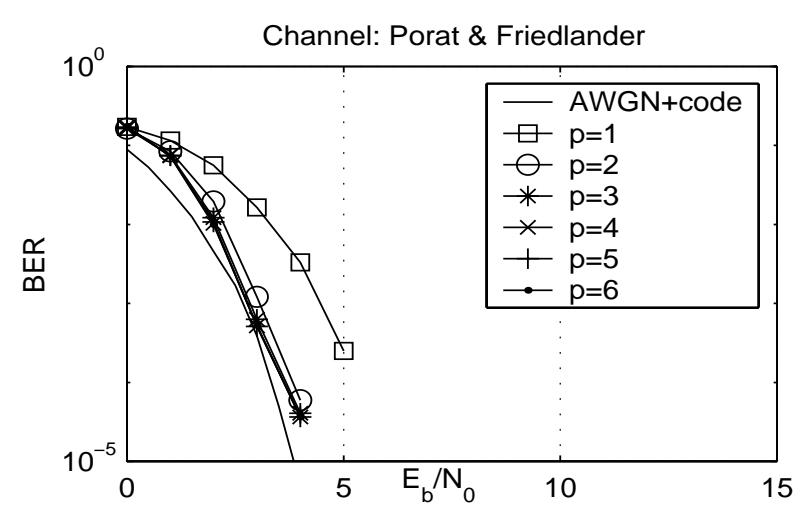

a. Simulated performance of the Turbo-equalizer

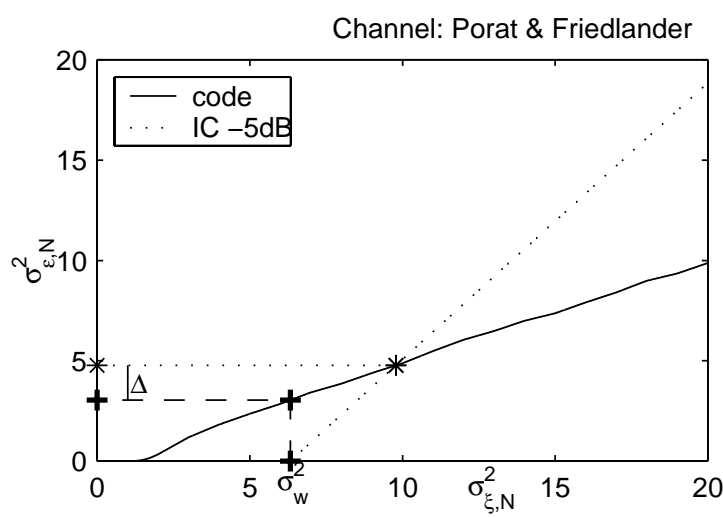

b. Convergence analysis.

Fig. 5. Porat and Friedlander's channel. 


\section{C. "Tough" channels}

In this section, we consider "tough" channels (Proakis B and C $\left[6\right.$, page 616]) such as the $\frac{\gamma_{h}(0)^{2}}{\Gamma_{h}}$ of which is respectively 1.06 and 0.49 . For these channels, the characteristic of the decoder during the Turbo simulation differs from the function $f$ simulated above for an AWGN channel. In these cases, we observed on simulation that the noise at the output of the IC is white but not gaussian, as indicated by the D'Agostino's test [3] based on third and fourth order statistics. This may explain why the decoder's performance is reduced. In spite of this mis-matched decoding, $\sigma_{\varepsilon, N}^{2}$ may be further used to predict the performance of the Turbo-equalizer (without carrying out the simulation) by penalizing the input variance of the decoder with the ratio between the minimal distances of the dispersive and of the AWGN channel:

$$
\sigma_{\varepsilon, N}^{2}=f\left(\sigma_{\xi, N}^{2} \frac{d_{\text {min dispersive channel }}^{2}}{d_{\text {min AWGN channel }}^{2}}\right) \text {, where } \frac{d_{\text {min dispersive channel }}^{2}}{d_{\text {min AWGN channel }}^{2}} \leq 1 \text {. }
$$

The accuracy of the prediction depends on how the penalized function matches the simulated ones (see Figure 6), as we see below.
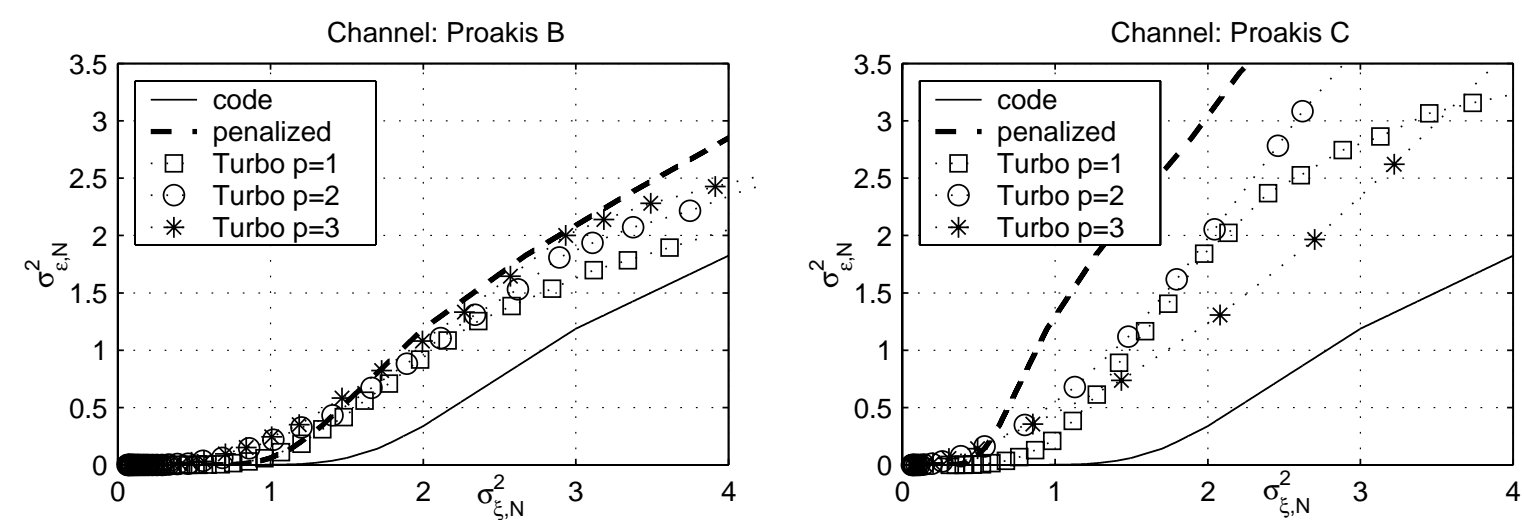

Fig. 6. Accuracy of the penalized curve of the decoder and the simulated ones.

Moreover, we have shown earlier [7] that there is a trigger point in the iterative process, followed by a breakdown effect. After the trigger point, the BER decreases steeply as a function of the decoding step $p$. The present analysis allows us to predict this trigger point.

The analysis for the Proakis B channel shows that the limit of the stability of the fixed point is close to the trigger point (1.5 to $2 \mathrm{~dB}$ for simulation to be compared with $3 \mathrm{~dB}$ for analysis, see Figure 7.a). Also shown is the prediction of the trigger point for channel Proakis C. For this channel, note that the fixed point does not always exist, depending on $\sigma_{w, N}^{2}$. The limit of existence of this fixed point occurs at $6.5 \mathrm{~dB}$ as is shown in Figure 7.b and matches reasonably well with the trigger point (6 to $10 \mathrm{~dB}$ for simulation, depending on the equalizer of the first iteration).

We focus now on the gap between the curves of the decoder fed by AWGN noise and by the output of the IC (see Figure 6). Next, we propose to further analyze this gap and compare it to other features of the channel such as its capacity. 


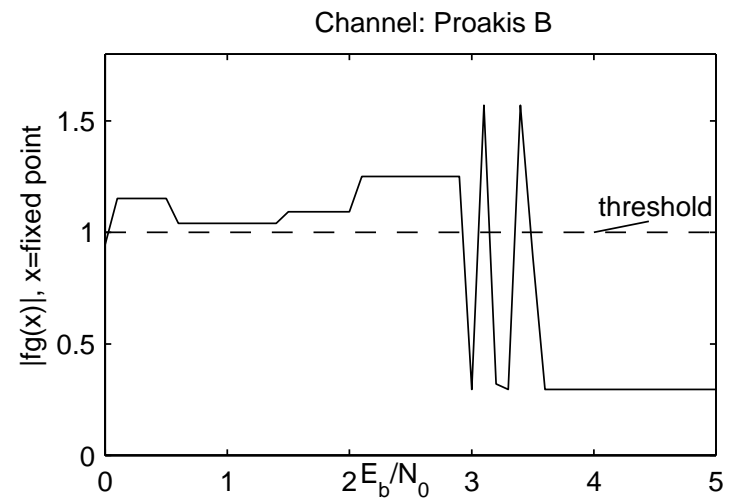

a. Proakis B: stability of the fixed point.

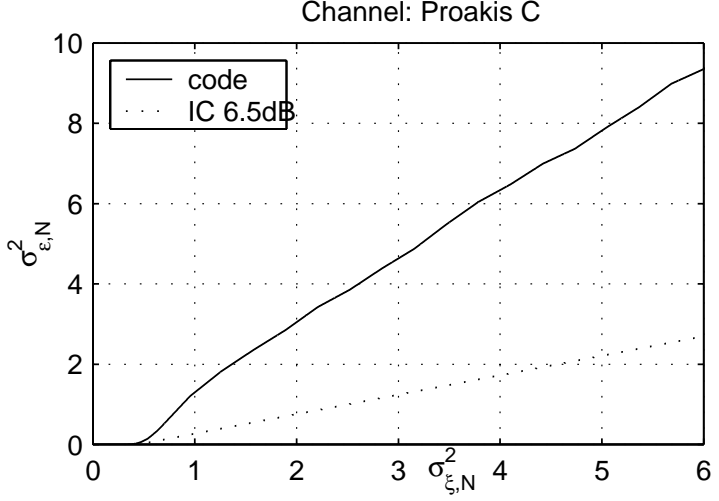

b. Proakis C: existence of the fixed point.

Fig. 7. Prediction of the trigger point.

\section{CONCLUSION}

We modeled here the distribution of the estimates of the transmitted symbol $d_{n}$ (given $d_{n}$ ) in the Turbo-equalizer by a white gaussian one. Using this model, we computed the error variances and the evolution of these variances through the iterative process, obtaining a convergence analysis. Because of mis-matched decoding during iterative process, we had to penalize the decoder with the ratio between the minimal distances of the dispersive and of the AWGN channel. This allowed us to predict the trigger point observed in Turbo-equalizer's performance. Depending on the channel, the prediction is based on either the limit of existence of the fixed point or the limit of stability of this point (if the fixed point exists). Based on this analysis, we propose a definition of a "tough" channel, when processed by the Turbo-equalizer.

\section{REFERENCES}

[1] P.D. Alexander, A.J. Grant, "Iterative channel and information sequence estimation in CDMA," IEEE Sixth International Symposium on Spread Spectrum Techniques \& Applications (ISSSTA'00), New Jersey, USA, Sept. 2000.

[2] L.R. Bahl, J. Cocke, F. Jelinek, J. Raviv, "Optimal decoding of linear codes for minimizing symbol error rate," IEEE Trans. Inf. Th., pp. 284-287, March 1974.

[3] R.B. D'Agostino, E.S. Pearson, "Test for departures from normality. Empirical results for the distributions of $b_{2}$ and

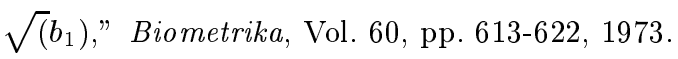

[4] A. Glavieux, C. Laot, J. Labat, "Turbo-equalization over a frequency selective channel," Int. Symp. on Turbo-codes, Brest, France, pp. 96-102, 1997.

[5] B. Porat, B. Friedlander, "Blind equalization of digital communications channels using high order moments," IEEE Trans. of Sig. Proc., Vol. 39, No. 2, pp. 522-526, Feb. 1991.

[6] J.G. Proakis, Digital Communications (3rd edition), McGraw-Hill, 1995.

[7] A. Roumy, I. Fijalkow, D. Pirez, "Joint equalization and decoding: why choose the iterative solution?," IEEE 50th Vehicular Technology Conference, pp. 2989-2993, Amsterdam, The Netherlands, September 1999. 